\title{
Acquired Chiari Malformation: Safety of Neuraxial Anesthesia?
}

\author{
Twinkal P. Dalal, Taruna Penmetcha, Maria Torres, Ramsis Ghaly
}

John H. Stroger Jr. Hospital of Cook County, Chicago, USA.

Email: twinkal.dalal@gmail.com

Received October $28^{\text {th }}, 2012$; revised November $29^{\text {th }}$, 2012; accepted December $29^{\text {th }}, 2012$

\begin{abstract}
Chiari malformation is a congenital anomaly that primarily involves the downward displacement of the cerebellar tonsils through the foramen magnum and elongation of forth ventricle and lower brainstem. Patients with Chiari I (congenital or acquired) malformation are asymptomatic or may present with neurologic signs and symptoms. It is always a question of safety of neuraxial anesthesia in these patients. There is potential risk of dural puncture that can initiate the neurologic symptoms or worsen the existing symptoms due to CSF leakage or tonsillar herniation. Other side, performance of neuraxial anesthesia can cause acquired Chiari I type malformation due to CSF leak and intracranial hypotension. We reviewed the case reports and articles regarding safety of neuraxial anesthesia in the setting of Congenital or Acquired Chiari malformation.
\end{abstract}

Keywords: Chiari Malformation; Neuraxial Anesthesia

\section{Introduction}

Chiari I malformation is a congenital anomaly that primarily involves the downward displacement of the cerebellar tonsils through the foramen magnum and elongation of forth ventricle and lower brainstem. It is commonly associated with underdevelopment of posterior cranial fossa with overcrowding of normally developed hindbrain. Chiari II, III and IV have also been described and they are scaled according to severity. Type I is common but less severe condition. Classes III and IV are rare but being severe. The term Arnold Chiari has fallen somewhat for type II Chiari malformation; some sources are still using that term for all four types of Chiari malformation. Other conditions are sometime associated with Chiari I malformation are hydrocephalus, syringomyelia, scoliosis, kyphosis, tethered spinal cord and also some connective tissue disorders.

The term "Acquired Chiari malformation" is sometimes applied to cerebellar tonsillar ectopia in the setting of spontaneous cerebrospinal fluid (CSF) leakage and resulting intracranial hypotension. Spontaneous CSF leakage is a well described disorder in the literature. CSF leakage can be iatrogenic occurrence also. It may occur after lumber puncture, myelography, spinal anesthesia, craniotomy or spinal surgery or may be caused by blunt or penetrating spinal trauma. There are case reports of occurring intracranial hypotension occurring after CSF diversion procedure through lumboperitoneal shunt.
Sathi et al. has reported a case of acquired Chiari malformation due to persistent CSF leak after multiple lumber puncture. C samil has reported a case of Pseudo chiari type I malformation secondary to CSF leakage after lumber spine surgery.

Patient with Chiari I (congenital or acquired) malformation are asymptomatic or may presents with neurologic signs and symptoms. It is always a question of safety of neuraxial anesthesia in these patients. There is potential risk of dural puncture can initiate the neurologic symptoms or worsen the existing symptoms due to CSF leakage or tonsillar herniation. On the other side, performance of neuraxial anesthesia can cause acquired Chiari I type malformation due to CSF leak and intracranial hypotension. We reviewed the case reports and articles regarding safety of neuraxial anesthesia.

\section{Epidemiology}

Prevalence of Chiari malformation (congenital or acquired) is 1 per 1000 to 1 per 5000. Type I Chiari malformation is common, less severe and treatable condition. Most of the patients are asymptomatic. Incidence of symptomatic Chiari are less and unknown.

\section{Signs and Symptoms}

Neurologic signs and symptoms associated with dysfunction of the cerebellum, brainstem and/or cervical spinal cord, usually arise during third decades of life. The signs 
and symptoms include sub occipital headaches (81\%), Ocular disturbances (78\%), otoneurological disturbances (74\%), lower cranial nerve, brain stem and cerebellar disturbances (52\%) and disturbances of spinal cord function. Obstructive hydrocephalus and blockade of CSF flow lead to syrinx formation, eventually leading syringomyelia. Rarely patient with central cord syndrome presents with hand muscle weakness, sensory disturbances and paralysis may occur. Spontaneous or iatrogenic cerebral hypotension due to CSF leak presents with postural symptoms.

\section{Diagnosis}

Chiari I malformation is determined by neuroimaging studies like MRI of head and cervical spine. Radiograph of cervical spine may reveal spondylosis or other associated abnormalities. Intracranial hypotension also determines by brain MRI, which shows any combination of brain sagging, pachymeningeal enhancement and subdural collections. CSF leak can be demonstrated on spinal MRI, myelography or cisternography.

\section{Discussion}

Chiari I malformation either congenital or acquired may be associated with herniation and compression of cerebellar tonsils and brainstem. Spinal anesthesia with CSF leak could create negative spinal pressure and worsening of herniation or compression, eventually worsening of neurologic symptoms. Similarly Epidural block could end up with wet tap and worsen the symptoms. There are couples of case reports out with worsening of symptoms after neuraxial block in Chiari I patient. It is also a dilemma to perform the blood patch after a wet tap in patient with Chiari I formation which could be acquired after CSF leak from wet tap.

So far three case reports suggest that dural puncture with Chiari I malformation might exacerbate symptoms. Hullander et al. reported a case of 31-year-old parturient developed recurrent headache after spinal block for cesarean delivery and an MRI scan revealed a Chiari I malformation [1]. Barton and Sharpe reported a case of late onset visual changes after wet tap during placement of lumber epidural in 33-year-old parturient. Her symptoms worsened over the year and Chiari I malformation was diagnosed on MRI scan. She was treated with sub occipital craniotomy [2]. Sathi et al. also reported a case of acquired Chiari I malformation after multiple lumber puncture [3].

Contrary, there are small studies and case reports are available suggesting safety of performing neuraxial block in Chiari I malformation. Chantigian et al. published a small series of 12 parturient with this malformation who delivered 30 babies, 9 were had neuraxial anesthesia.
None of these patients who had neuraxial anesthesia for their delivery developed symptoms or had worsening of preexisting symptoms of Chiari I malformatation. There was only one patient who received continuous spinal block developed postdural puncture headache that resolved after blood patch. None of the patient in the series had symptoms of severely elevated ICP. They recommended that it is safe to perform the regional anesthesia in Chiari I patient after proper evaluation regarding signs of elevated intracranial pressure or significant neurologic deficit in which cases neuraxial block is contraindicated. General anesthesia could elevated ICP during laryngoscopy and can worsen the symptoms; one should always weigh risk and benefits of all form of anesthesia [4]. Margarido et al. published a case report of post traumatic cervical syringomyelia presented for cesarean delivery. MRI of the spine revealed a syrinx of $2 \mathrm{~mm}$ in diameter extending from C4 - T1. There was no sign of elevated ICP. An epidural catheter was safely placed with ultrasound guidance without complications [5].

Acquired Chiari I malformation may occur after lumber puncture or unintentional wet tap after epidural anesthesia. It may occur after spontaneous leakage of CSF associated with intracranial hypotension. Atkinson et al. published a seven cases of Acquired Chiari I malformation secondary to spontaneous CSF leakage and chronic intracranial hypotension [6]. Difference from idiopathic Chiari I malformation is chronicity of symptoms and contrast MRI reveals diffuse patchy meningeal enhancement in case of intracranial hypotension. Treatment of the Acquired Chiari due to spontaneous intracranial hypotension is blood patch at the leak site. Surgical correction only requires if blood patch fails. Another case report by Kranz et al. showed resolution of syringohydromyelia associated with intracranial hypotension with targeted CT guided epidural blood patch [7]. These reports are suggesting epidural blood patch for treatment of spontaneous CSF leak and Acquired Chiari malformation. Similarly it can occur after spinal anesthesia or wet tap after epidural block, it may treat with epidural blood patch.

\section{Conclusion}

We are suggesting that neuraxial anesthesia is not contraindicated in case of congenital Chiari I malformation if patient does not have any signs of elevated ICP or significant neurologic deficit. General anesthesia can elevate the ICP and worsen the symptoms. One should always weight risk and benefits of different anesthesia. Acquired Chiari I malformation may occur after neuraxial block due to CSF leak. Epidural blood patch should be indicated in that case to seal the leak side, suggesting blood patch should perform under CT guidance or ultra- 
sound technique to prevent subsequent wet tap which may worsen the symptoms.

\section{REFERENCES}

[1] R. M. Hullander, T. D. Bogard, D. Leivers, D. Moran and D. M. Dewan, "Chiari I Malformation Presenting as Recurrent Spinal Headache,” Anesthesia \& Analgesia, Vol. 75, No. 6, 1992, pp. 1025-1026. doi:10.1213/00000539-199212000-00026

[2] J. J. Barton and J. A. Sharpe, “Oscillopsia and Horizontal Nystagmus with Accelerating Slow Phases Following Lumbar Puncture in the Arnold-Chiari Malformation," Annals of Neurology, Vol. 33, No. 4, 1993, pp. 418-421. doi:10.1002/ana.410330418

[3] S. Sathi and P. E. Stieg, “Acquired Chiari I Malformation after Multiple Lumbar Punctures: Case Report,” Neurosurgery, Vol. 32, No. 2, 1993, pp. 306-309.

[4] R. C. Chantigian, M. A. Koehn, K. D. Ramin and M. A.
Warner, “Chiari I Malformation in Parturients,” Journal of Clinical Anesthesia, Vol. 14, No. 3, 2002, pp. 201-205. doi:10.1016/S0952-8180(02)00342-2

[5] C. Margarido, R. Mikhael, A. Salman and M. Balki, "Epidural Anesthesia for Cesarean Delivery in a Patient with Post-Traumatic Cervical Syringomyelia," Canadian Journal of Anesthesia, Vol. 58, No. 8, pp. 764-768. doi:10.1007/s12630-011-9525-3

[6] J. L. Atkinson, B. G. Weinshenker, G. M. Miller, D. G. Piepgras and B. Mokri, “Acquired Chiari I Malformation Secondary to Spontaneous Spinal Cerebrospinal Fluid Leakage and Chronic Intracranial Hypotension Syndrome in Seven Cases,” Journal of Neurosurgery, Vol. 88, No. 2, 1998, pp. 237-242. doi:10.3171/jns.1998.88.2.0237

[7] P. G. Kranz, R. J. Viola and L. Gray, "Resolution of Syringohydromyelia with Targeted CT-Guided Epidural Blood Patching,” Journal of Neurosurgery, Vol. 115, No. 3, pp. 641-644. doi:10.3171/2011.3.JNS102164 